Keio J. Med. 23: 205-210, 1974

\title{
STUDIES ON THE HEAD INJURIES OF HUMAN SUBJECTS IN ADVANCED AGE
}

(II)

\author{
NOBUO YOSHII*, KINYA OSHIDA** and SAKAE FUKUDA*** \\ * Neurological Surgery, Second Department of Surgery, Toho University, \\ School of Medicine, Tokyo \\ ** Neurosurgery, Saitama National Hospital, Wako City \\ ** Neurosurgery, Shizuoka Red Cross Hospital, Shizuoka City
}

(Received for publication Aug. 28, 1974)

\begin{abstract}
Examined the EEG evaluation results of 218 aged patients over 60 years old with head injuries, in order to explore the relationship between the EEG evaluation results and the period of unconsciousness, period from injuries to the EEG test, skeletal bone fracture, and remarkable clinical symptoms. As a result, it was found out that: 1) The older the age, the higher the frequency of abnormal EEG, 2) The longer the period of unconsciousness, the higher the frequency of abnormal EEG, while the longer the period of unconsciousness, the longer the period of abnormal EEG after injuries, 3) Those with skull fracture and/or remarkable clinical symptoms indicated higher frequency of abnormal EEG. Brains of aged people are prone to indicate highly notable changes once external forces are applied to them, and this is attributed to the changes usually taking place as the age advances, unlike the changes indicated by the immature brains of infants. Hence, clinical application of EEG should by done with consideration paid in connection with precise clinical examinations. It should be emphasized that enough care should be taken in its evaluation by performing repeated EEG tests with some intervals.
\end{abstract}

Traffic accidents have remarkably increased in recent years as the number of automobiles increased very sharply. It is claimed that $700,000-800,000$ people are being affected by traffic accidents each year. Of these, 500,000 (approximately $70 \%$ ) are affected by head injuries. Such accidents in recent years are partly featured by the sharp rise in the head injuries of aged people due to extended working age of people. Aged people, however, differ from younger people both mentally and physically including the cerebral conditions, so that their head injuries develop likewise different ensuing features. The brains of aged people are 
under physiological and sometimes pathological retrograde changes, so that it would be a problem what changes in the brains of aged people are like, once they are affected by external forces. We have evaluated through EEG the changes of brains of aged people developed after head injuries. It seems that there have been hardly any reports handling EEG changes of aged people with head injuries, although those with regular adults are available in a large number.

\section{CASE AND METHOD}

Patients we treated amounted to 218 who were selected from among the ambulatory patients older than 60 having paid their first visits to Neurosurgical Clinic for out patients of Keio University Hospital during the period from January 1963 to February 1968 for treatments with EEG examinations, by excluding those whose clinical findings were insufficiently recorded, who showed unconsciousness at testing, who were soon after their operations, or who were under treatment with oral administration of CNS-tropic agents. The patients comprised $171(78.4 \%)$ in their $60 \mathrm{~s}, 39(17.9 \%)$ in their $70 \mathrm{~s}$, and $8(3.7 \%)$ in their age over 80 .

Thirteen channel EEG machine (a product of Nippon Koden Co., Ltd.) and disk electrode of silver-silver chloride for EEG test were employed. Electrodes were arranged at 18 locations including frontal, precentral, central, parietal, occipital, anterior temporal, middle temporal, and postrior temporal region of both hemispheres and both ear lobes. The recording was made with all the subjects at rest using unipolar and bipolar tracing for 20-40 minutes, together with hyperventilation and photic stimulation.

Evaluation was made with these patients concerning the time of unconsciousness, the period from infliction of head injuries to the EEG test, skull fracture, and relationship between the clinical findings and EEG results. If patients were subjected to repeated EEG tests, the results of the initial test were adopted for evaluation.

\section{RESULTS}

\section{EEG Evaluation:}

The results of EEG evaluation comprised 91 normal $(41.7 \%), 26$ on the borderline $(11.9 \%)$, and 101 abnormal $(46.3 \%)$. The frequency of the abnormal cases was found to be higher as the age of the patients advanced.

2. Unconsciousness and EEG Evaluation Results:

The results of examination on the unconsciousness were classified into the 
following seven groups: 1) Those with no clouding of consciousness whatsoever, 2) Those with unconsciousness lasting for less than 10 minutes, 3) Those with unconsciousness lasting for less than one hour, 4) Those with unconsciousness lasting for less than 6 hours, 5) Those with unconsciousness lasting for less than 24 hours, 6) Those with unconsciousness lasting for more than 24 hours, 7) Those with unconsciousness lasting for unknown period.

Those who had no clouding of consciousness at the time of injuries amounted to 108 cases ( $49.5 \%$ ), while those who had unconsciousness more or less amounted to 88 cases $(40.3 \%)$, and those whose details of unconsciousness were unavailable amounted to 22 cases $(10.1 \%)$. The relationship between the unconsciousness and EEG evaluation results turned out that normal EEG results shared $51.9 \%$ in the cases with negative unconsciousness, while abnormal EEG results shared $31.5 \%$, and that the normal shared $29.6 \%$ in the cases with positive unconsciousness, while abnormal EEG results shared $62.5 \%$. The relationship between the time length of unconsciousness and EEG evaluation results proved that abnormal EEG results were more frequent among those with unconsciousness for more than one hour and the frequency of abnormal EEG results tended to be higher as the period of unconsciousness was longer (Fig. 1).

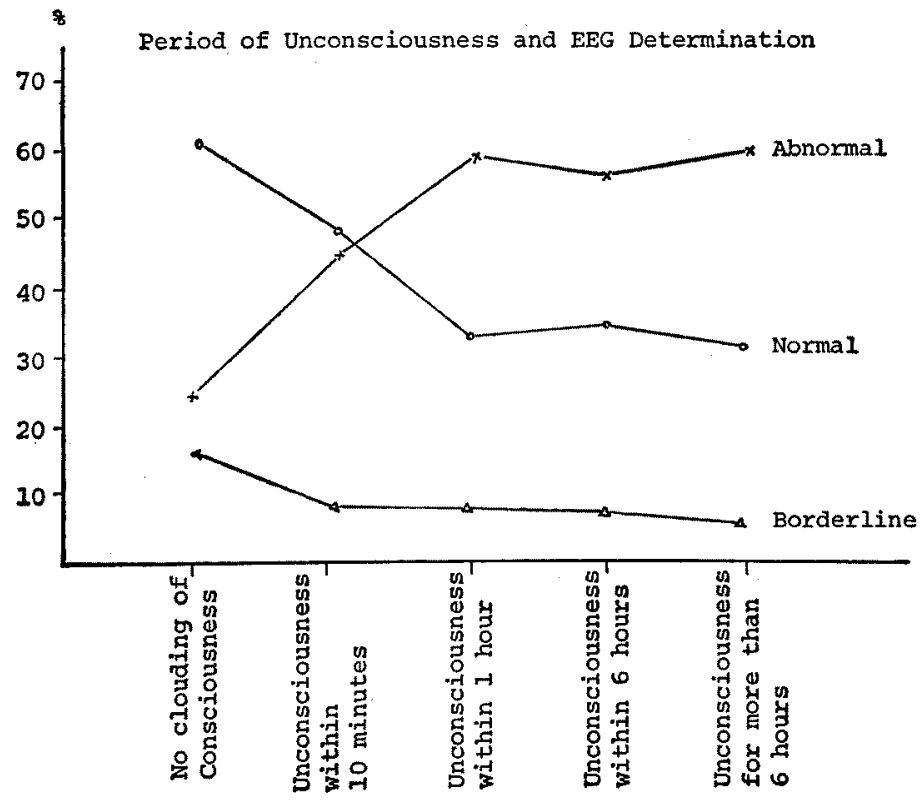

Fig. 1 
3. Period from Injuries to EEG Examination and EEG Evaluation Results:

The majority of the patients underwent the initial test within 10 months after their injuries.

The results of evaluation on the relationship between the EEG results and the period from the injuries to the EEG examination proved that those with longer period from the injuries to the test tended to show higher frequency of abnormalities in their EEG results (Fig. 2).

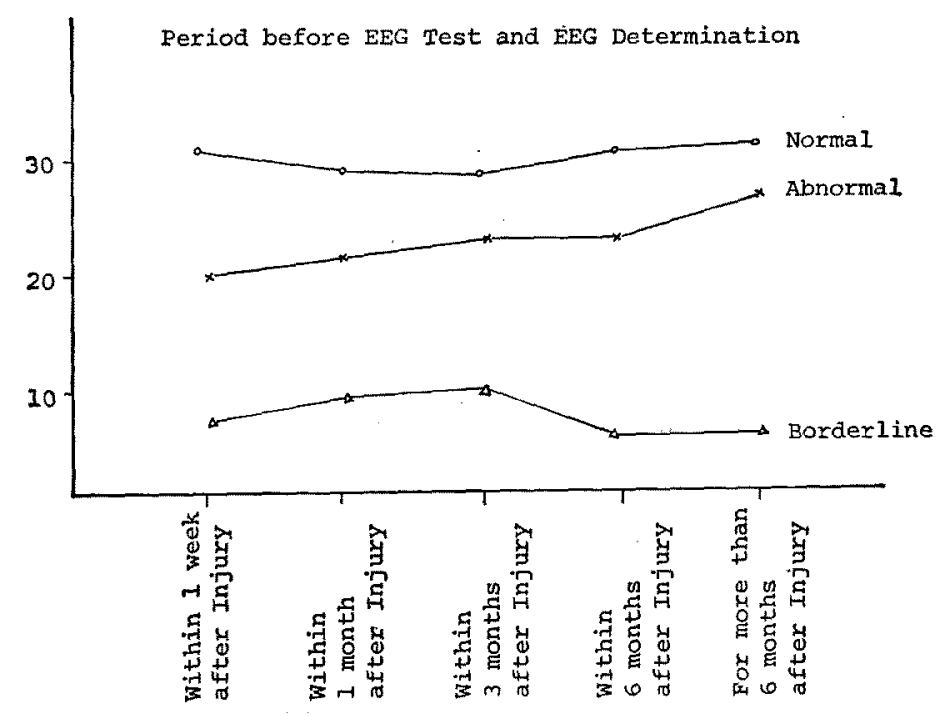

Fig. 2

\section{Skull Fracture and EEG Evaluation Results:}

Thirteen patients were confirmed through roentogenographic examination to have been affected by skull fracture. The results of their EEG evaluation proved that three of them were normal, whereas one was on the borderline and the balance nine indicated abnormalities. In other words, $76.9 \%$ indicated more or less abnormal findings, including the one on the borderline.

5. Clinical Findings and EEG Evaluation Results:

Twelve patients developed apparent clinical symptoms including paralysis of extremities, dysesthesia, speech disturbances, convulsive seizures, and psychic symptoms. Of these, $8(66.7 \%)$ indicated abnormal EEG and balance 2 each were with borderline EEG and normal one, respectively. In other words, the more notable their clinical symptoms were, the higher was the frequency of abnormalities. 


\section{DISCUSSION}

EEG varies with the advancement of age. Silverman ${ }^{2}$ reported that, from the evaluation results of adults, abnormal pattern tended to appear in EEG more frequently as the age of patients advanced, quoting the results of 55\% of patients in their $60 \mathrm{~s}$ and $54 \%$ of those in their 70s, having normal EEG, but only $33 \%$ of those in their age over 80 . Busse et al ${ }^{2}$ reported that $85 \%$ of normal young human subjects in their age of 20-39 indicated normal EEG results, while those in age of 40-59 showed $65 \%$ and the aged people in age of 60-79 showed $51 \%$, indicating the trend of degreasing frequency of normal EEG results. Ohtomo ${ }^{3}$ reported that abnormal EEG was seen in $32.7 \%$ of aged people in normal conditions in their age of over 60 , and that the frequency of abnormalities was found higher as the age of the people advanced. All of these reports handled patients hospitalized in sanatoriums and hospitals, whereas Yoshii ${ }^{4}$ reported that the results of evaluation of EEG of $\mathbf{2 0 5}$ aged people over their age of $\mathbf{5 0}$ in their normal social activities proved presence of $19 \%$ abnormal EEG, $19.5 \%$ on the borderline, and $61.5 \%$ normal findings. If these are to be limited to those over 60,29 $(25.8 \%)$ indicated abnormal records, while $21(18.7 \%)$ were on the borderline and $62(55.5 \%)$ showed no abnormalities. The frequency of development of abnormal records in those with no clouding of consciousness in our cases was nearly the same as this, whereas the frequency of abnormal records in the patients with unconsciousness was obviously higher than in the normal aged subjects.

It is of interest that, although the frequency of abnormalities in EEG of aged patients with head injuries is high as stated above, the abnormalities were likewise more frequent and longer among those who had longer period of unconsciousness, which would be indicative of the big influences of such great external forces as would cause prolonged unconsciousness upon the brains of aged people. More specifically, the progressive changes due to advancement of age which are latent in the brains of aged people would not only magnify the changes in EEG together with the influences of external forces, but also retard the recovery of these people from the influences.

In evaluating the frequency of these abnormal EEG tracing, not only the changes in EEG due to advancement of age, but also the period from the traumata to the EEG examination would be important. EEG test soon after the injuries with unconsciousness indicates abnormalities in EEG with no exception, and it takes considerably long period to recover from the abnormalities. It is claimed that the sooner the EEG test is done after the injuries, the more notable is the degree of abnormalities in EEG. Since our patients were mostly those treated on out patient clinic, there were few patients in their acute stage; the soonest was 7 days after the trauma before our EEG test, so that it can be likely for this reason 
that the frequency of abnormal EEG was lower in our patients. It should be attended to that the longer the period after thei njuries the higher was the frequency of abnormal EEG. Since the presence of skull fracture can be deemed as representative of external strength, it would be a matter of course that the frequency of abnormal EEG was high.

Of the 12 cases indicating marked clinical symptoms at the time of the EEG test, 2 indicated normal EEG. Williams ${ }^{5}$ reported that there would be hardly any improvements in clinical symptoms of patients indicating such findings as this.

Detailed analysis of clinical findings on the injured locations and the classes of traumata thus examined and detailed investigations on EEG findings would have to be further explored and looked into, but these will be done in other reports in the near future.

\section{REFERENCES}

1. Silverman, A. J., Buses, E. W. and Barnes, R. H.: Studies in the process of aging; Electroencephalographic findings in 400 elderly subjects, Electroencephalogr. Clin. Neurophysiol. 7: 67, 1955

2. Busse, E. W. and Obrist, W. D.: Presenescent Electroencephalographic Changes in Normal Subjects. J. Gerontol. 20: 213, 1965

3. Ohtomo, E., Kameyama, M. and Tsubaki, T.: Studies on EEG of Senile Patients (Part 1). Rinsho Yakuri 4: 573, 1964

4. Yoshii, N.: Electroencephalographic Observation of Apparently Normal Aged Subjects. Tohoku J. exp. Med. 103: 203, 1971

5. Williams, D.: The Electroencephalogram in Chronic Post Traumatic States. J. Neurol. Psychiat. 4: 131, 1941 